\title{
Prevalence of severe Plasmodium knowlesi infection and risk factors related to severe complications compared with non-severe $P$. knowlesi and severe $P$. falciparum malaria: a systematic review and meta-analysis
}

\author{
Manas Kotepui ${ }^{* *}$ Kwuntida Uthaisar Kotepui ${ }^{1}$, Giovanni D. Milanez ${ }^{2}$ and Frederick R. Masangkay ${ }^{2}$
}

\begin{abstract}
Background: Plasmodium knowlesi is a potential cause of severe and fatal malaria, but comprehensive studies of its pooled prevalence and risk factors are lacking. This study aimed to explore the prevalence and risk factors related to severe $P$. knowlesi infection.

Methods: A systematic review was conducted by retrieving all published articles on severe $P$. knowlesi available in Web of Science (ISI), Scopus, and PubMed (MEDLINE). Titles, abstracts, and full-text articles were screened, and any irrelevant studies were excluded. The random-effects model was used to compute the pooled prevalence estimate of severe $P$. knowlesi infection by a metaprop command provided in STATA software. Differences in demographic characteristics, clinical characteristics, and laboratory data were analysed using Review Manager Version 5.3 software for patients in the following groups: 1) patients with severe and non-severe $P$. knowlesi infection and 2) patients with severe $P$. knowlesi and severe P. falciparum infection.

Results: Out of the 2382 studies retrieved from the three databases, seven studies with a total enrolment of 1124 patients with $P$. knowlesi infections were eligible to be included in this systematic review and meta-analysis. The pooled prevalence estimate of severe $P$. knowlesi infection was 19\% (95\% Cl: $\left.11-27 \%, l^{2}=93.7 \%\right)$. Severe acute kidney injuries (AKI) (77 cases, 45.6\%), jaundice (71 cases, 42\%), and hyperparasitaemia (55 cases, 32.5\%) were the common clinical manifestations found among patients with severe complications. In comparison to non-severe $P$. knowlesi infections, patients with severe $P$. knowlesi infections had significantly higher age, leucocyte count, and parasitaemia levels $(P<0.05)$. In comparison to patients with severe $P$. falciparum infections, patients with severe $P$. knowlesi infections had significantly higher age, neutrophil count, and creatinine levels $(P<0.05)$.

(Continued on next page)
\end{abstract}

\footnotetext{
* Correspondence: manas.ko@wu.ac.th

${ }^{1}$ Medical Technology, School of Allied Health Sciences, Walailak University,

Tha Sala, Nakhon Si Thammarat, Thailand

Full list of author information is available at the end of the article
}

(C) The Author(s). 2020 Open Access This article is licensed under a Creative Commons Attribution 4.0 International License, which permits use, sharing, adaptation, distribution and reproduction in any medium or format, as long as you give appropriate credit to the original author(s) and the source, provide a link to the Creative Commons licence, and indicate if changes were made. The images or other third party material in this article are included in the article's Creative Commons licence, unless indicated otherwise in a credit line to the material. If material is not included in the article's Creative Commons licence and your intended use is not permitted by statutory regulation or exceeds the permitted use, you will need to obtain permission directly from the copyright holder. To view a copy of this licence, visit http://creativecommons.org/licenses/by/4.0/ The Creative Commons Public Domain Dedication waiver (http://creativecommons.org/publicdomain/zero/1.0/) applies to the data made available in this article, unless otherwise stated in a credit line to the data. 
(Continued from previous page)

Conclusions: This systematic review and meta-analysis demonstrated a high proportion of severe $P$. knowlesi infections. Patients with severe $P$. knowlesi infections had higher age, leucocyte count, and parasitaemia levels than those with nonsevere $P$. knowlesi infections. In addition, patients with severe $P$. knowlesi infections had higher age, neutrophil count, and creatinine levels than those with severe $P$. falciparum infections.

Keywords: Plasmodium knowlesi, Severe malaria, Plasmodium falciparum, Prevalence

\section{Background}

Plasmodium knowlesi is a naturally occurring malarial parasite in monkeys [1] and is acknowledged as the fifth human malarial parasite, especially among travellers returning from Southeast Asian countries or among people living in endemic areas where monkeys are present, such as in Malaysian Borneo [2]. The natural reservoir hosts of $P$. knowlesi are the long-tailed macaque (Macaca fascicularis) and the pig-tailed macaque (Macaca nemestrina, Macaca leonine) [3-6]. The geographical distribution of P. knowlesi is found only in Southeast Asian countries because its natural hosts and the female Anopheles mosquito vectors are very limited and specifically exist in these areas [4]. A previous study demonstrated that the mosquito vectors in the Leucosphyrus Complex transmitted P. knowlesi in southern Asia, while the Dirus Complex transmitted P. knowlesi in Vietnam, Myanmar, Laos, Cambodia and Thailand [6, 7]. At present, there is no evidence indicating that $P$. knowlesi is transmitted from human to human but rather through mosquito bites [8]. Infections of $P$. knowlesi occur through quotidian (24-hour schizogonic cycles) malarial parasites and lead to severe and lethal manifestations.

The first case of $P$. knowlesi infection in humans was reported by Singh et al. in Sarawak, Malaysia, in patients previously misdiagnosed with $P$. malariae infections [2]. The morphology of $P$. knowlesi is similar to that of $P$. malariae, a quartan malarial parasite causing non-severe human malaria [9]. This similarity contributes to the problem that technicians encounter when identifying species of malaria under light microscopy. The evidence was supported by a study by William et al., which demonstrated that some infections with P. knowlesi confirmed by polymerase chain reaction (PCR) were miss-detected as $P$. malariae by microscopic methods (76\%) [10]. The rapid diagnostic tests (RDTs) were commercially available for the detection of $P$. knowlesi, but they were insensitive in cases of low parasitaemia and were unable to distinguish $P$. knowlesi from $P$. vivax $[11,12]$. Hence, confirmation of $P$. knowlesi requires a molecular technique, such as the nested PCR assay. A previous study demonstrated that $P$. knowlesi misdiagnosis as $P$. malariae caused severe malaria and resulted in death because of inappropriate treatment [13]. It is very important to emphasize to physicians the need to recognize nonfalciparum malaria as a potential cause of severe and fatal malaria in addition to $P$. vivax and to prevent the fatal outcome of patients by administering intravenous therapy with artesunate or quinine as the first drug of choice for severe malaria, especially caused by P. knowlesi. Although P. knowlesi is a potential cause of severe and fatal malaria, comprehensive studies of its pooled prevalence and risk factors are lacking. In addition, a meta-analysis determining the difference in the prevalence estimate of severe $P$. knowlesi infection among studies conducted on P. knowlesi is needed. Therefore, this study aimed to estimate the prevalence and risk factors related to severe $P$. knowlesi malaria. In addition, the comparison of demographic characteristics, clinical data, and laboratory data between severe $P$. knowlesi and severe $P$. falciparum infection was evaluated to predict disease severity in coendemic primary care settings of $P$. knowlesi.

\section{Methods}

\section{Article searching strategy}

This systematic review and meta-analysis was performed according to the Preferred Reporting Items for Systematic Review and Meta-Analysis (PRISMA) guidelines (PRISMA 2009 Checklist S1). Web of Science (ISI), Scopus, and PubMed were queried for studies before March 16, 2019, using the terms "(severe OR complicated OR complication) AND (("Plasmodium knowlesi") OR knowlesi)" (Table S1). This search was limited to human studies in the English language. The population, intervention, control, and outcomes (PICO) format was used to define the selection criteria: P, patients with severe P. knowlesi; I, severe malaria; $C$, patients with severe $P$. falciparum; and $\mathrm{O}$, demographic characteristics, clinical characteristics, and laboratory profiles. The present study included crosssectional studies that reported on the prevalence of $P$. knowlesi malaria in humans. Studies were excluded if they were case reports or case series, animal studies, clinical drug studies, co-infection studies, commentaries, experimental studies, reviews, or reports with incomplete data. Additionally, any study using the same data reported by groups of authors was excluded from the present study. 


\section{Data extraction}

Two reviewers (MK and KUK) selected and extracted the data from the included studies independently. We used the Endnote software X7 (Clarivate Analytics, Philadelphia, USA) to manage the references. First, we removed any duplicate papers retrieved from the three databases. Then, we screened the titles and abstracts according to the inclusion criteria. Finally, we assessed all full-text articles for detailed assessments for further data extraction. The data extraction was conducted using the Microsoft Excel 2010 (Microsoft Corporation, Washington, USA) and included data on the primary author, year of publication, study area, study design, demographic characteristics of patients (age and sex), and clinical characteristics (duration of fever, signs and symptoms, temperature in degrees Celsius $\left[{ }^{\circ} \mathrm{C}\right]$, blood pressure, pulse rate, respiratory rate, and previous malaria exposure). Laboratory profiles including parasite count, haemoglobin, leucocyte count, platelet count, creatinine, sodium, bilirubin, glucose, albumin, aspartate aminotransferase (AST), and alanine aminotransferase (ALT) were additionally collected. The malaria endemicity of the years when the included studies were conducted by their authors was demonstrated by the slide positivity rate as reported by the World Malaria Report 2010-2014 [14-18]. Disagreements in data extraction were resolved through discussion or by consulting a third reviewer (FRM).

\section{Criteria of severe $P$. knowlesi malaria}

The criteria of severe P. knowlesi malaria were defined by the World Health Organization (WHO) [19].

\section{Characteristics of the included studies}

A quality assessment was performed following the quality assessment tool developed by the Newcastle-Ottawa Scale (NOS) for assessing the quality of nonrandomized studies in meta-analysis [20]. It provided a star system with a maximum of nine stars to judge the included studies on three broad perspectives: the selection of the study groups, the comparability of the groups, and the ascertainment of outcome among the studies.

\section{Data synthesis}

The primary outcome of the present study was the pooled prevalence of severe $P$. knowlesi infection. The data analysis for the pooled prevalence began with entry of the extracted data into the Excel sheet and to the STATA Statistical Software version 15.0 (StataCorp LLC, Texas, USA). The number of patients with severe $P$. knowlesi and total cases of P. knowlesi were imported and analysed using a command "metaprop case population, random/fixed" provided in the STATA software. A metaprop command allowed Freeman-Tukey double arcsine transformation to stabilize the variances. The random-effects or fixed-effect model could be used to compute the pooled estimate, depending on the heterogeneity among the included studies [21]. The results are presented with the pooled prevalence along with the 95\% confidence interval $(C I)$.

The secondary outcome of the present study was to identify factors associated with severe $P$. knowlesi malaria using the demographic characteristics, clinical characteristics, and laboratory profiles of patients with severe and non-severe $P$. knowlesi malaria. The third outcome of the present study was to compare the demographic characteristics, clinical characteristics, and laboratory profiles between patients with severe $P$. knowlesi and severe $P$. falciparum malaria. The results of the second and third outcomes were presented as the pooled mean difference (MD) with $95 \%$ CI for continuous outcomes and odds ratio along with 95\% CI for categorical outcomes. The data heterogeneity was assessed using Cochrane Q (chi-square) and Moran's $I^{2}$ (inconsistency). The $I^{2}$ was quantified as low, moderate, and high, with upper limits of 25,50 , and $75 \%$ for $I^{2}$, respectively. If moderate or high heterogeneity was presented by Moran's $I^{2}$, the random-effects model was used to calculate the pooled estimates. If a low heterogeneity $\left(I^{2}<25 \%\right)$ was presented, the fixed-effect model was used to calculate the pooled estimates. The subgroup analysis of study sites was performed to demonstrate whether the transmission intensity between Sarawak and Sabah, the area with the highest transmission intensity of all malaria cases and the area with the highest cases of $P$. knowlesi in Malaysia during 2013-2017, affected the pooled prevalence estimate or affected the risk of severe $P$. knowlesi infection in the meta-analysis. The analysis of the second and third outcomes was performed using the Review Manager 5.3 (The Cochrane Collaboration, London, UK). The meta-regression analysis of a covariate, namely, malaria endemicity (slide positive rate), between severe $P$. knowlesi and non-severe P. knowlesi was performed using STATA Statistical Software version 15.0.

\section{Publication bias}

The funnel plot was used to demonstrate any symmetrical funnel plot among patients with severe P. knowlesi infection. The evaluation of publication bias among the included studies helped explore the underlying cause of data heterogeneity if it was present in the included studies.

\section{Results}

Search results and study characteristics

A total of 2382 studies were retrieved from PubMed $(n=117)$, Scopus $(n=2139)$, and Web of Science $(n=$ 126) databases (Fig. 1), of which 208 studies were found to be duplicates. Of the 2174 articles reviewed, 2056 articles were excluded because they were not related to $P$. 


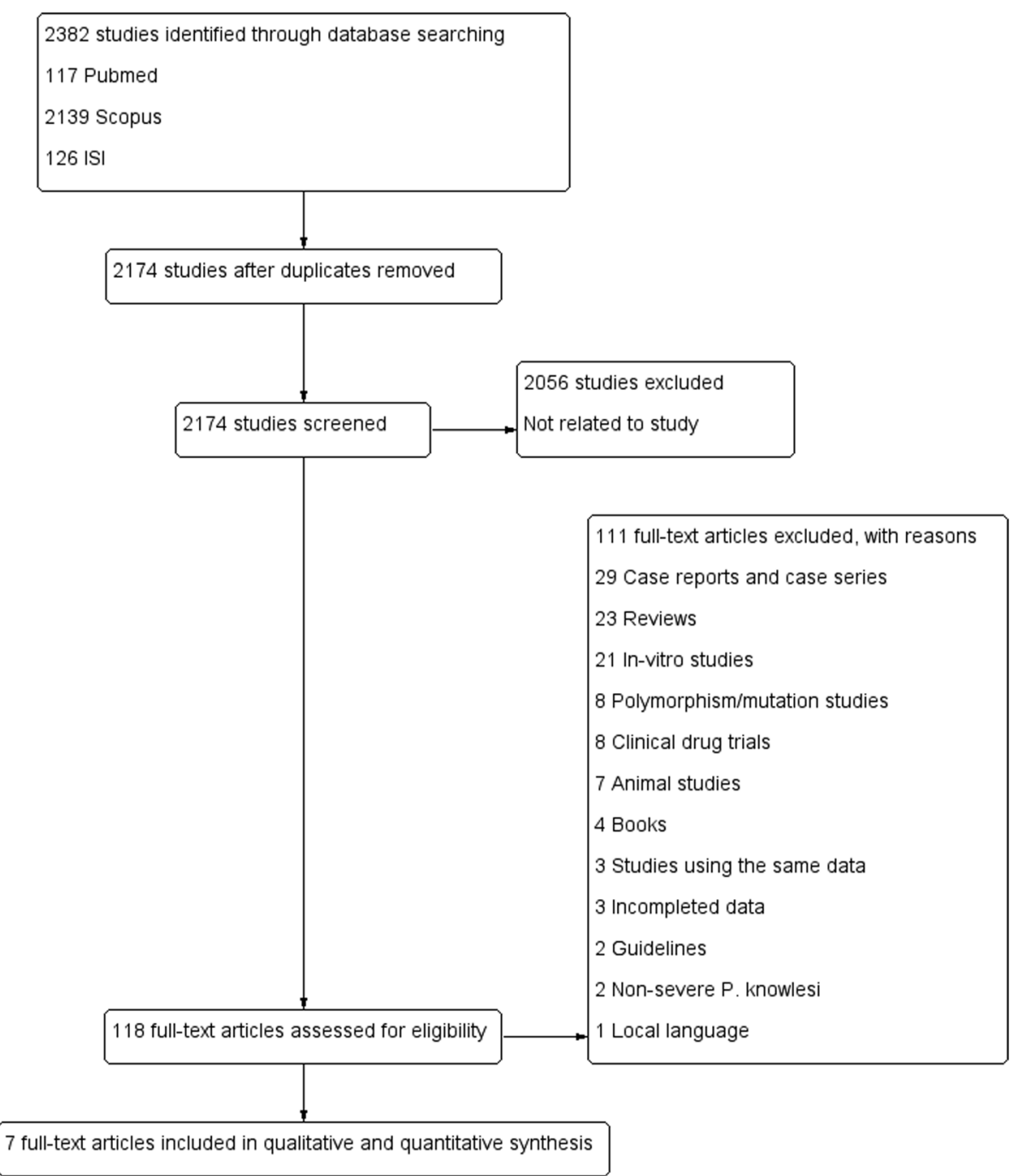

Fig. 1 PRISMA diagram. Flow chart for study selection

knowlesi. Of the 118 full-text articles reviewed, 111 articles were excluded for various reasons, as shown in Fig. 1. Seven studies were included for systematic review and meta-analysis.

The characteristics of the seven studies included in this study are presented in Table 1, Table S2, and Table S3. Table 1 presents demographic profiles and the number of patients with $P$. knowlesi and P. falciparum infections. Of those seven studies, all cases were conducted in Malaysia during 2006-2013. Four studies [22, 26, 28, 29] were conducted in Sabah, whereas the other three studies were conducted in Sarawak [23, 24, 27], Malaysian Borneo. The seven studies included prospective observational studies [22, 28, 29], cross-sectional observational studies [23, 24], a retrospective study [26], and a case control study [27]. All seven studies reported a total of 1124 patients with $P$. knowlesi infections. Of those 1124 patients, 169 patients (15\%) had severe complications. Most of the severe complications found in patients included severe acute kidney injury (AKI) (77 cases, 45.6\%), jaundice (71 cases, $42 \%$ ), and hyperparasitaemia (55 cases, 32.5\%). The number of patients with $P$. falciparum infections included a total of 394 patients in five studies [22-24, 28, 29]. Of those 394 patients, 44 patients (11.2\%) had severe complications. Common clinical manifestations found in patients included jaundice (24 cases, 54.5\%), shock (15 cases, $34.1 \%$ ), and respiratory distress (11 cases, 25\%). Other complications of severe P. knowlesi and P. falciparum infections are shown in Table 1.

\section{Prevalence of severe $P$. knowlesi malaria}

The pooled prevalence of severe $P$. knowlesi malaria was 19\% (95\% CI: $\left.11-27, I^{2}=93.7 \%\right)$, calculated by a random-effects model since high heterogeneity (Moran's $I^{2}$ : 93.7\%) was found (Fig. 2). The highest prevalence of severe $P$. knowlesi malaria was reported in studies by 


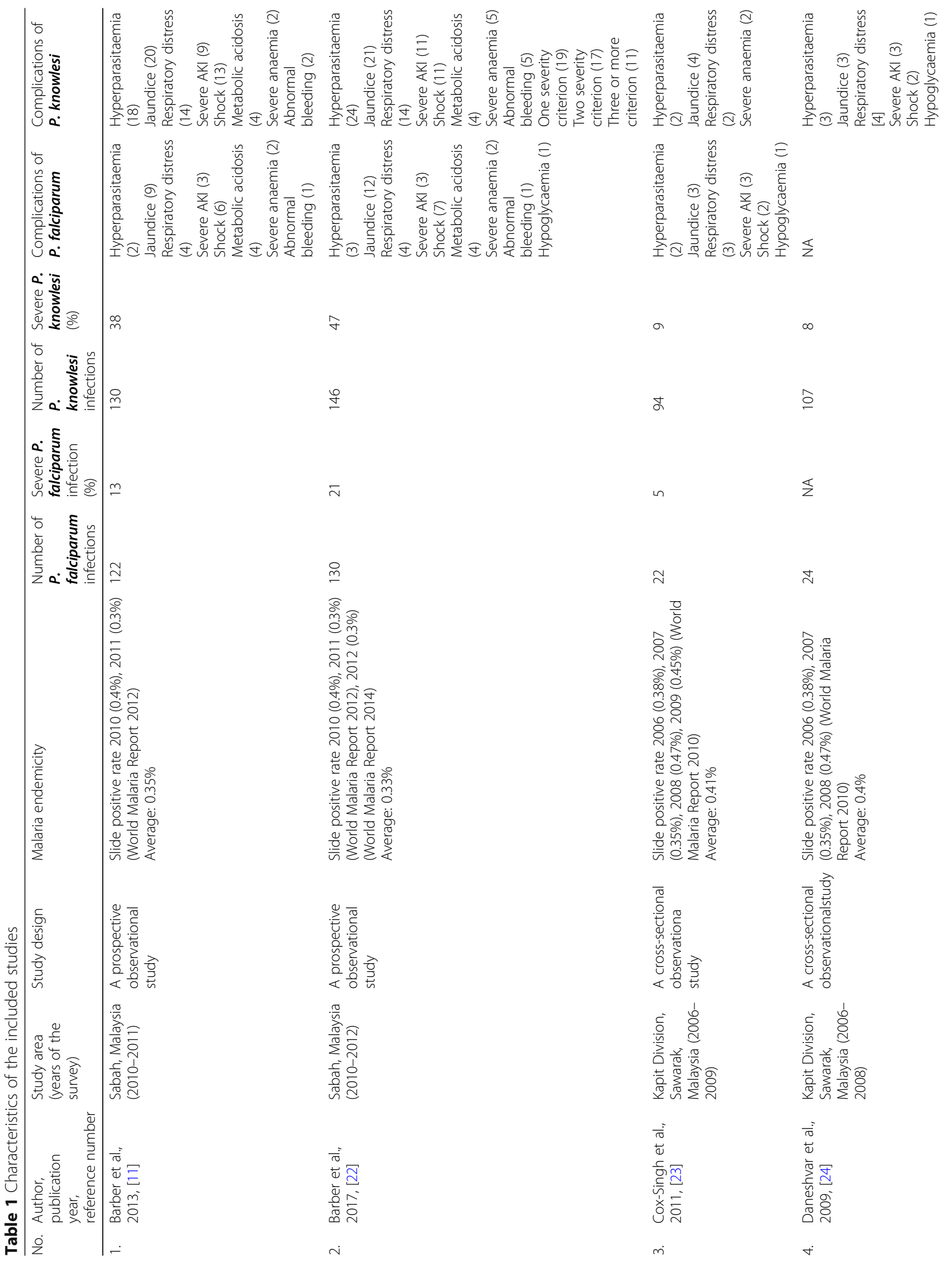




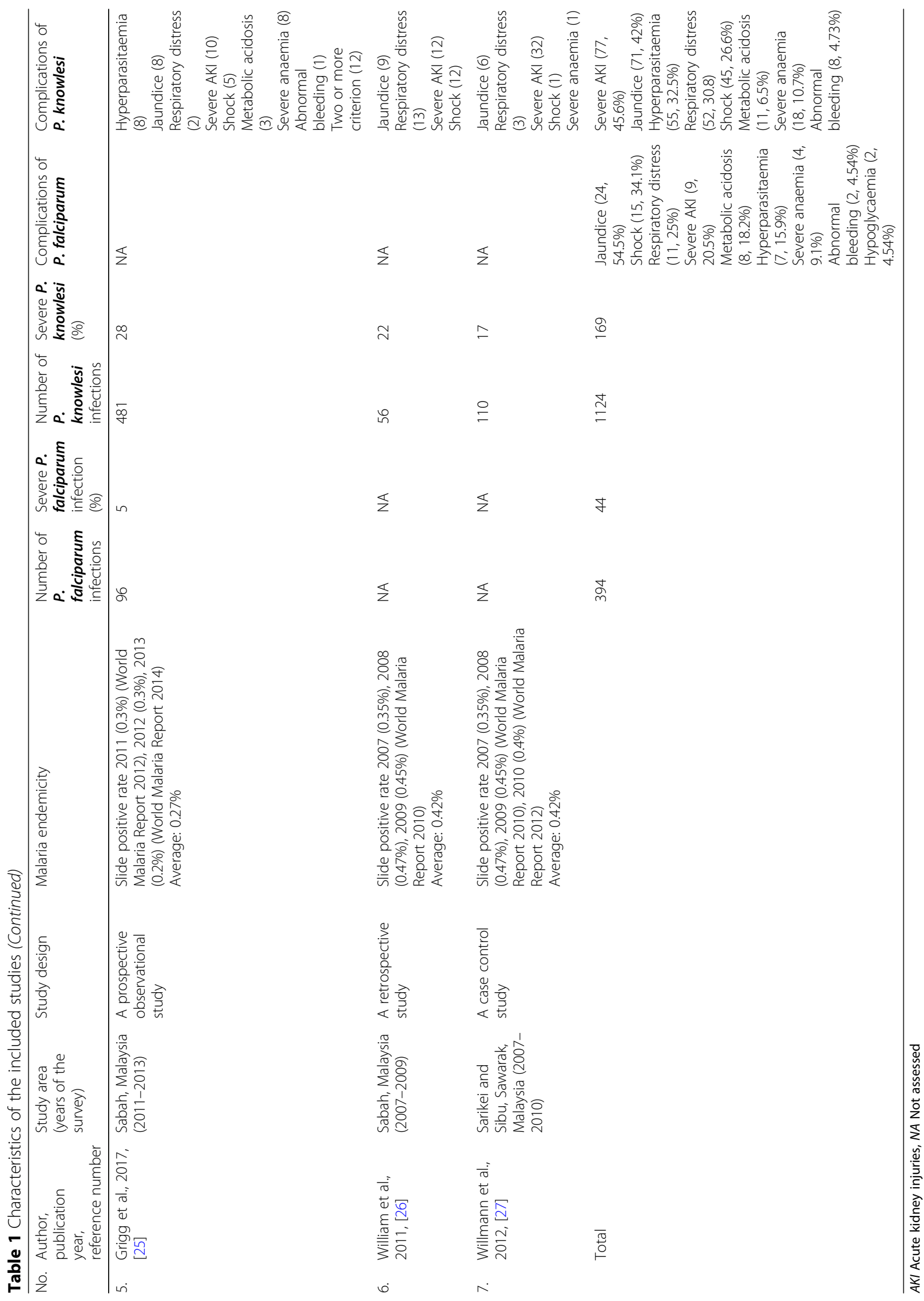


William et al. (39, 95\% CI: 28-52\%) [26] and by Barber et al. (32, 95\% CI: $\left.25-40, I^{2}=93.7 \%\right)$ [22], while the lowest prevalence of severe $P$. knowlesi malaria was reported in a study by Grigg et al. (6, 95\% CI: 4-8\%) [29]. The subgroup analysis of study sites demonstrated that the pooled prevalence estimate of the included studies conducted in Sabah (26, 95\% CI: 8-44\%, $\left.I^{2}=96.7 \%\right)$ was higher than that of the studies conducted in Sarawak (10, 95\% CI: 6-15\%, $\left.I^{2}=43 \%\right)$.

\section{Risk factors associated with severe $P$. knowlesi malaria}

Cochrane Q and Moran's $I^{2}$ analysis of age $\left(I^{2}=66 \%\right)$, leucocyte count $\left(I^{2}=54 \%\right)$, and parasitaemia level $\left(I^{2}=\right.$ 91\%) between patients with severe and non-severe $P$. knowlesi infections demonstrated that medium and high heterogeneities were found among the included studies; therefore, the random-effects model was used to calculate the pooled MD along with 95\% CI. In comparison to patients with non-severe $P$. knowlesi infections, the results demonstrated that patients with severe $P$. knowlesi infections were significantly older than patients with non-severe $P$. knowlesi infections $(P<0.0001, \mathrm{MD}$ : 10.08, 95\% CI: 5.04-15.11, $I^{2}=66 \%$ ) (Fig. 3). The subgroup analysis of study sites demonstrated that a significantly older age was found in both the included studies conducted in Sabah $(P=0.03$, MD: 10.75 , 95\% CI: 3.75$\left.17.75, I^{2}=42 \%\right)$ and those studies conducted in Sarawak $\left(P=0.04\right.$, MD: $\left.10.08,95 \% C I: 0.51-20.27, I^{2}=81\right)$. The meta-regression analysis using the slide positive rate as a covariate revealed that the slide positive rate was not a confounding factor for the age difference $(P=0.718, t=$ 0.42, 95\% CI: - 10.8-13.1).

Patients with severe $P$. knowlesi infections had a significantly higher leucocyte count than patients with nonsevere $P$. knowlesi infections $(P=0.0004, M D: 2440,95 \%$ $C I$ : $1080-3810, I^{2}=88 \%$ ) (Fig. 4). The subgroup analysis of study sites demonstrated that a significantly higher leucocyte count was found in the included studies conducted in Sarawak ( $P<0.00001$, MD: 2610, 95\% CI: 1580 $3650, I^{2}=53 \%$ ) but not in the included studies conducted

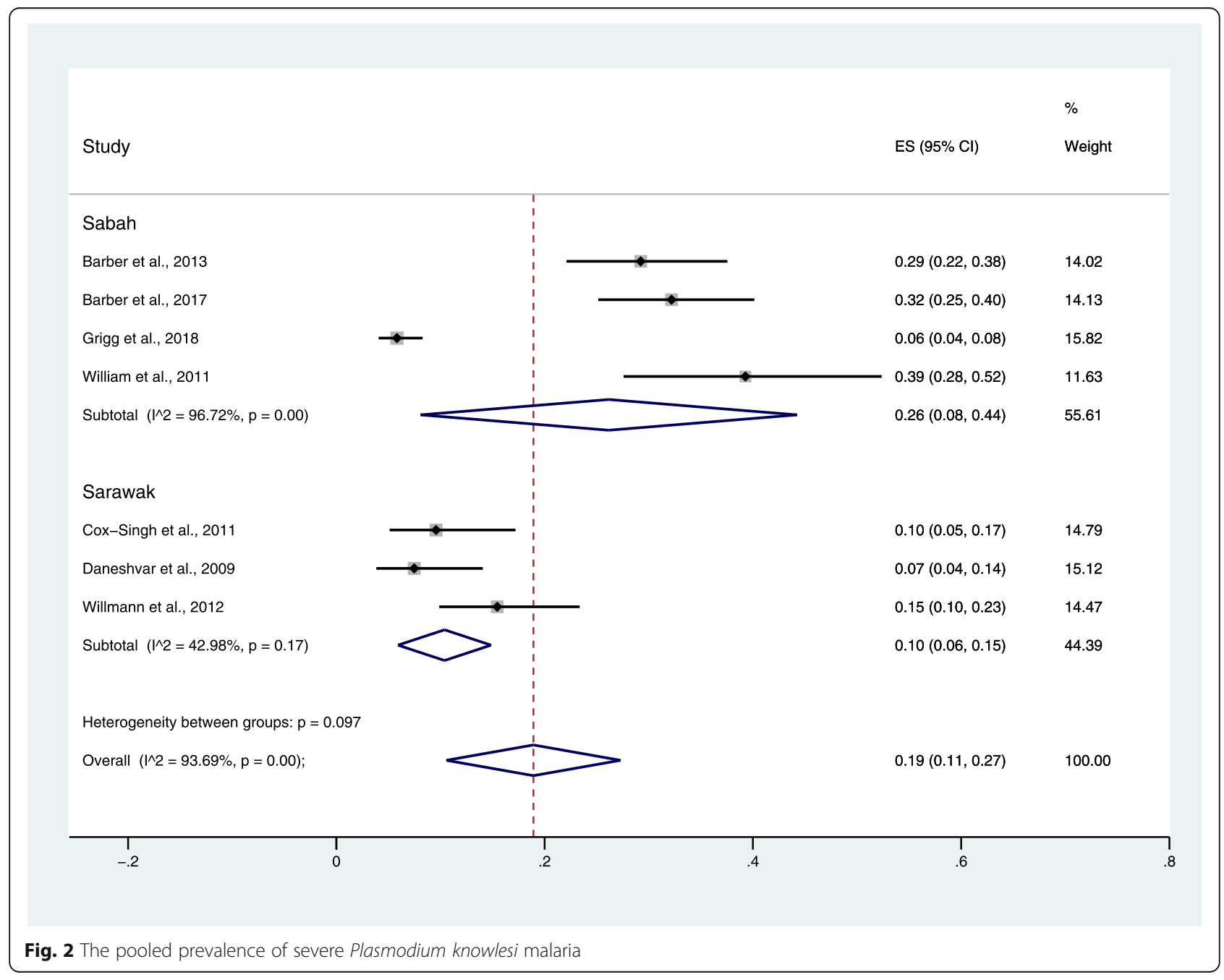




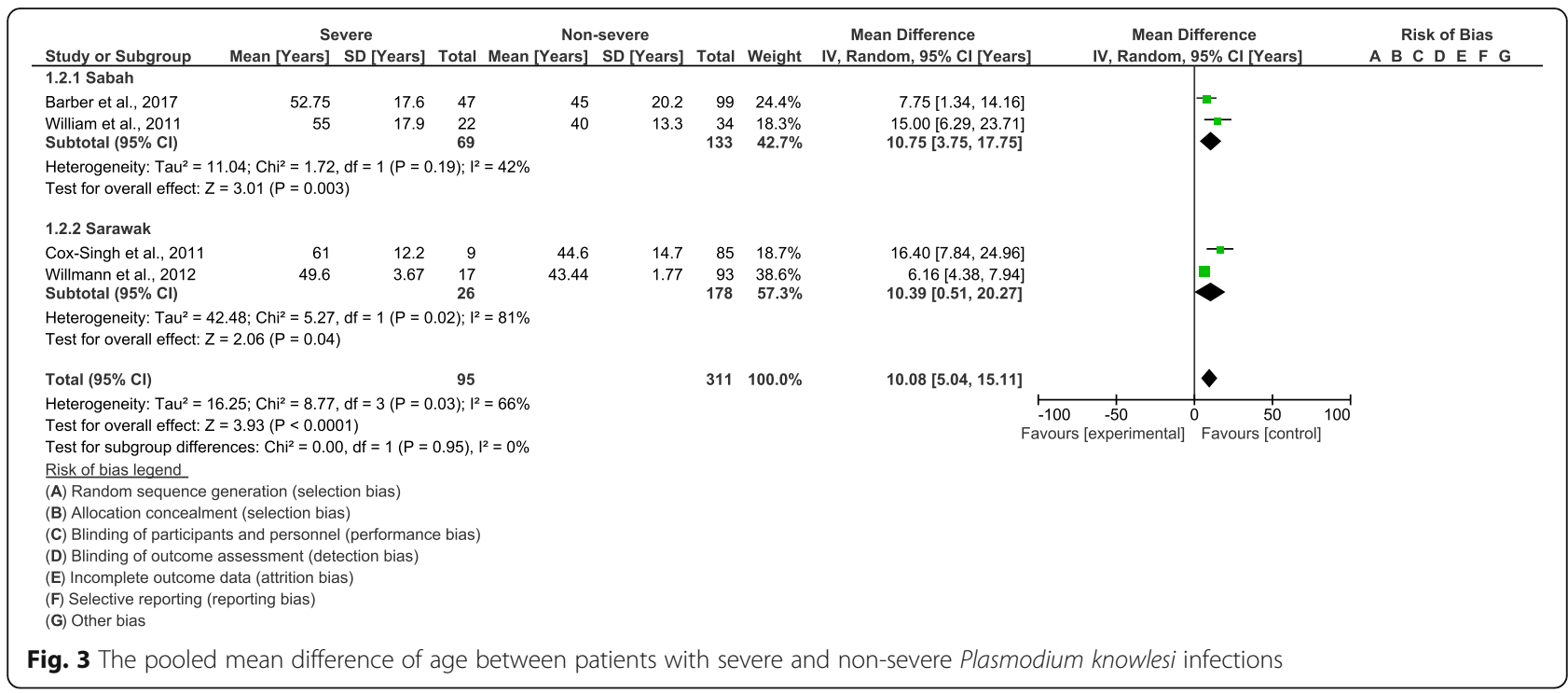

in Sabah $\left(P=0.14\right.$, MD: 2720, 95\% CI: $-880-6310, I^{2}=$ $88 \%)$. The meta-regression analysis using the slide positive rate as a covariate revealed that the slide positive rate was not a confounding factor for the leucocyte count difference $(P=0.516, t=0.78,95 \% C I$ : $-63-91)$.

Patients with severe $P$. knowlesi infections had a significantly higher parasitaemia level than patients with nonsevere $P$. knowlesi infections ( $P=0.007$, MD: 66 800, 95\% CI: 18340-115260) (Fig. 5). The subgroup analysis of study sites demonstrated that no subgroup difference was observed $(P=0.75)$, and the subgroup analysis demonstrated that no significantly higher parasitaemia level was found in the included studies conducted in Sarawak $(P=$ 0.06, MD: $76780,95 \%$ CI: $\left.-1760-155320, I^{2}=92 \%\right)$ compared to the included studies conducted in Sabah $(P=$ 0.06, MD: $60660,95 \% C I$ : $\left.-2550-123880, I^{2}=99 \%\right)$. The meta-regression analysis using the slide positive rate as a covariate revealed that the slide positive rate was not a confounding factor for the parasitaemia level difference $(P=0.375, t=-1.04,95 \%$ CI: $-60.7-30.8)$.
Cochrane Q and Moran's $I^{2}$ analysis of platelet counts between patients with severe and non-severe $P$. knowlesi infections demonstrated a high level of heterogeneity $\left(I^{2}=89 \%\right)$; therefore, the random-effects model was used to calculate the pooled MD along with $95 \% \mathrm{CI}$. In comparison to patients with non-severe $P$. knowlesi infections, patients with severe $P$. knowlesi infections had a significantly lower platelet count $(P<0.00001, \mathrm{MD}:-26$ 980, 95\% CI: - 34 370--19590) (Fig. 6). The subgroup analysis of study sites demonstrated that a subgroup difference was observed $(P=0.004)$, and a significantly lower platelet count was found in both the included studies conducted in Sabah $(P<0.00001, \mathrm{MD}:-21280$, 95\% CI: $\left.-28120-14440, I^{2}=74 \%\right)$ and those studies conducted in Sarawak $(P<0.00001$, MD: $-32550,95 \%$ $\left.C I:-36220-28890, I^{2}=0 \%\right)$. The meta-regression analysis using the slide positive rate as a covariate revealed that the slide positive rate was not a confounding factor for the platelet count difference $(P=0.848, t=-0.21$, 95\% CI: $-10.2-22)$.

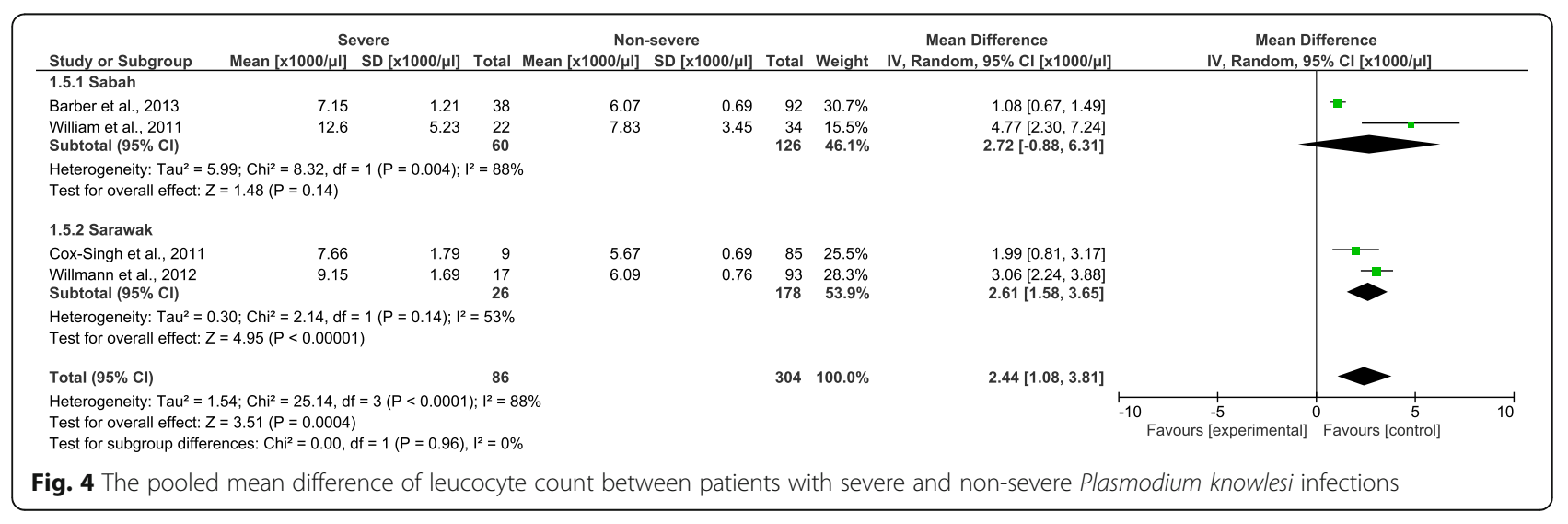




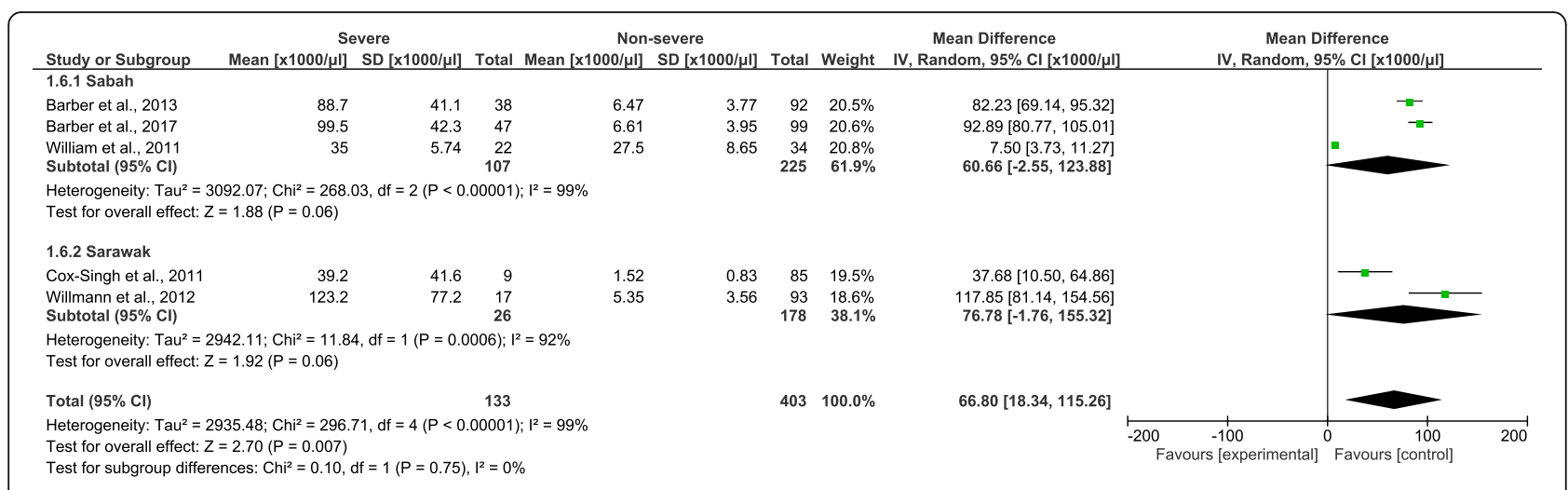

Fig. 5 The pooled mean difference of parasitaemia level between patients with severe and non-severe Plasmodium knowlesi infections

Severe $P$. knowlesi and severe $P$. falciparum malaria

Cochrane Q and Moran's $I^{2}$ analysis of age $\left(I^{2}=92 \%\right)$, neutrophil count $\left(I^{2}=66 \%\right)$, and creatinine level $\left(I^{2}=89 \%\right)$ between patients with severe $P$. knowlesi and severe $P$. falciparum infections demonstrated that medium and high heterogeneities were found among the included studies; therefore, the random-effects model was used to calculate the pooled MD along with $95 \%$ CI. In comparison to patients with severe $P$. falciparum infections, patients with severe $P$. knowlesi infections were older $(P<0.0001, \mathrm{MD}$ : 27.6, 95\% CI: 13.7-41.4) (Fig. 7), had a higher neutrophil count $(P<0.0001$, MD: 1420, 95\% CI: 730-2100) (Fig. 8 ), and had a higher creatinine level ( $P=0.01$, MD: 50.9, 95\% CI: 11.3-90.4) (Fig. 9). The meta-regression analysis using the slide positive rate as a covariate revealed that the slide positive rate was not a confounding factor for age $(P=$ 0.297, $t=-1.40,95 \% C I:-139.8-71.3)$, neutrophil count $(P=0.363, t=-1.56,95 \% C I:-169.9-132.7)$, or creatinine level $(P=0.289, t=2.05$, 95\% CI: $-100.8-139.6)$.

\section{Occupational risk of patients with $P$. knowlesi infections}

The proportion of farmers infected with $P$. knowlesi was not different from those infected with $P$. falciparum
$(P=0.19, O R=5.54,95 \% C I: 0.43-71.7)$ (Supplementary Figure 1). The proportion of plantation workers infected with $P$. knowlesi was not different from those infected with $P$. falciparum $(P=0.33, O R=0.19,95 \%$ CI: $0.01-$ 5.33) (Supplementary Figure 2).

\section{Quality of included studies and publication bias}

All seven studies included in the present study had max scores of nine stars according to the NOS guidelines, indicating good quality. The funnel plot analysis among the included studies could not be performed as it required a minimum of 10 studies for the analysis [30].

\section{Discussion}

This study provided prevalence data and risk factors related to severe $P$. knowlesi malaria in comparison with severe $P$. falciparum malaria. All seven included studies conducted in Malaysia reported on severe P. knowlesi complications. The subgroup analysis of study sites demonstrated that the pooled prevalence estimate of the included studies conducted in Sabah (26\%) was higher than that of the studies conducted in Sarawak (10\%). Previous studies reported that $P$. knowlesi, not $P$. 


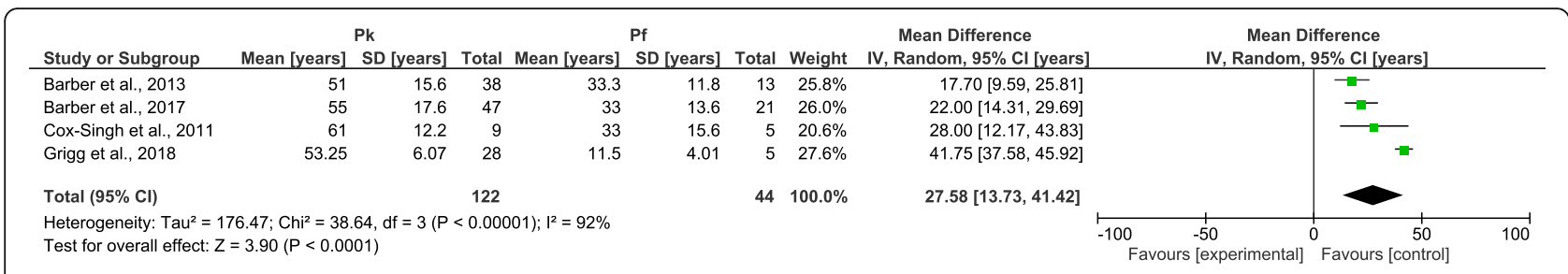

Fig. 7 The pooled mean difference of age between patients with severe Plasmodium knowlesi and severe P. falciparum infections

falciparum or $P$. vivax, was the most common cause of malaria in Malaysia, particularly in Sabah and Sarawak $[31,32]$. Another study revealed that the highest prevalence of $P$. knowlesi malaria was observed in wild macaques (87\%) and long-tailed macaques (50\%) living in Sarawak, Malaysian Borneo [4, 33]. The most recent retrospective study, which reviewed the malariaconfirmed cases of the Ministry of Health, Malaysia between January 2013 and December 2017, revealed that the highest average incidence rate (AIR) was found in Sarawak (0.420 per 1000 population) and Sabah (0.383 per 1000 population) from 2013 to 2017 [34]. Our results suggested that although the prevalence of $P$. knowlesi was higher in Sarawak, the highest proportion of severe malaria caused by $P$. knowlesi was found in another state, Sabah. The result of our study was supported by the average mortality rate per 100000 patients with $P$. knowlesi being higher in Sarawak (0.074) than in Sabah (0.064) [34].

In addition to being endemic in Malaysia, the presence of P. knowlesi in wild long-tailed macaques (19\%) was also reported from the island of Palawan, the Philippines [35]. In humans, asymptomatic infections with P. knowlesi parasites were also found in individuals living close to forested areas in Cambodia (0.59\%) [36]. A previous study suggested that the microscopic appearance of $P$. malariae should be reported as $P$. knowlesi to increase recognition and be treated with intravenous artesunate for severe P. knowlesi malaria [37]. In Malaysia, current $P$. knowlesi malaria management guidelines recommend an initial treatment with intravenous artesunate for any patients $>50$ years of age or with a parasitaemia level $>$ $20000 / \mu \mathrm{l}[28,37]$.
The most common complications of severe P. knowlesi malaria found in the present study were severe AKI, jaundice, and hyperparasitaemia. The results were similar to the largest study of $P$. knowlesi malaria conducted in three hospitals in Malaysia, where AKI was frequently observed [29]. The mechanisms regarding the pathology and physiology of severe malaria from $P$. knowlesi in humans are not well understood. However, even a low parasite density from $P$. knowlesi infections can lead to severe diseases [16]. In severe P. knowlesi malaria, hyperparasitaemia has been defined as a parasite count of 100000 parasites/ $\mu$ l or higher $[28,38]$. The life cycle of $P$. knowlesi in the human host is a 24-hour life cycle in which it rapidly reproduces at the asexual stage at a high parasitaemia level more than P. falciparum [28]. For AKI, the mechanism in severe malaria from P. knowlesi is still unknown; however, microvascular sequestration similar to that in P. falciparum infections might be the cause that plays a role in kidney injury [39].

The meta-analysis demonstrated that older patients had more severe complications from $P$. knowlesi infections than younger patients, which was related to the low risk of anaemia and high risk of thrombocytopenia $[2,28,29]$. However, a previous study suggested that $P$. knowlesi infected a wide range of ages from young teenagers to mature adults, which was in accordance with a study in Sarawak, Malaysia [24]. Two previous studies demonstrated that the mean age of patients was 45 years $[24,28]$. A possible reason that might explain why $P$. knowlesi infections occurred more frequently among older patients was the strong correlation of increasing age with increasing parasitaemia [28]. Increasing age was associated with increasing systemic inflammation and

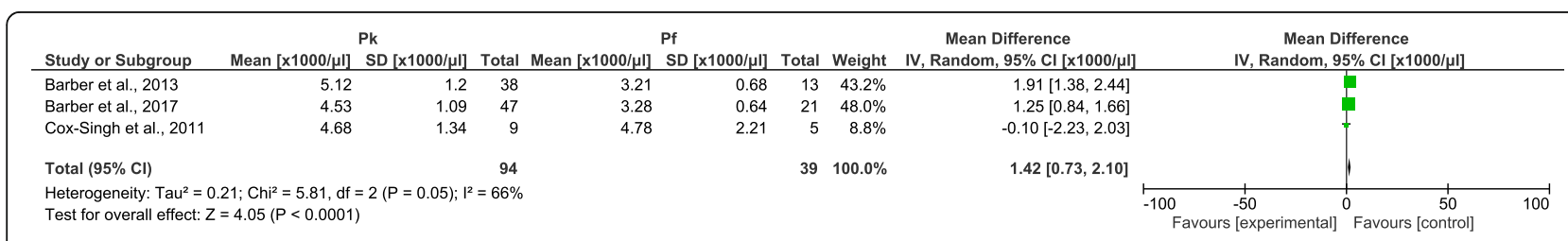

Fig. 8 The pooled mean difference of neutrophil counts between patients with severe Plasmodium knowlesi and severe $P$. falciparum infections 


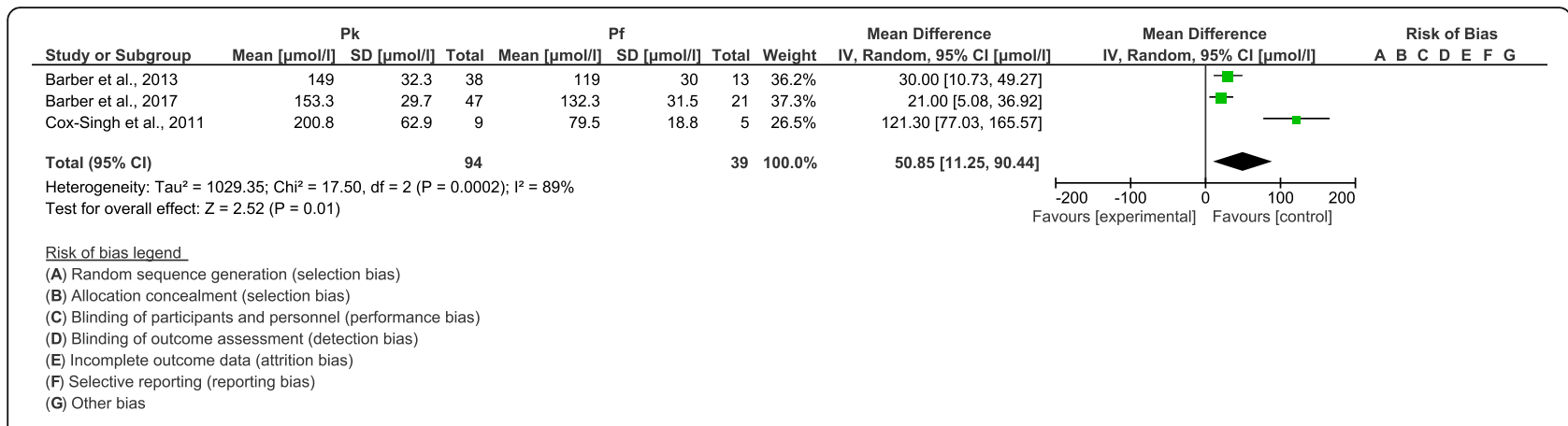

Fig. 9 The pooled mean difference of creatinine levels between patients with severe Plasmodium knowlesi and severe P. falciparum infections

impairment of microvascular function, contributing to severe malaria in older adults with $P$. knowlesi infections [22]. This mechanism-related severe malaria in older patients compared to younger patients might be similar to that in P. falciparum malaria, where endothelial activation increases with age [40]. In addition, the limited outdoor activities of children may be related to the low evidence of $P$. knowlesi infections among these age groups [32]. A previous study also suggested that the transmission of $P$. knowlesi over a wide age range might be related to the presence of family clusters [41]. The results of the present study demonstrated that the proportion of occupations (farmers and plantation workers) was similar in both $P$. knowlesi and P. falciparum infections. This demonstrated that patients who worked as farmers and plantation workers could be infected with either P. knowlesi or P. falciparum at the same proportion. However, a previous study indicated that $P$. knowlesi parasitaemia was significantly higher in farmers and plantation workers than in other occupations [22]. Moreover, farming was a risk factor for the infection of $P$. knowlesi malaria but not for non-P. knowlesi malaria [25]. Previous studies demonstrated that the risk of $P$. knowlesi infection was related to human behaviours, such as higher forest exposure or working in agricultural areas $[25,28]$. A previous study also showed that males demonstrated a four-fold greater risk of symptomatic $P$ knowlesi infection and that the risk of infection by $P$. knowlesi and non-P. knowlesi was comparable in males [25]. In contrast, sex was not significantly associated with $P$. knowlesi seropositivity in a study by Fornace et al., 2018, who conducted a study in northern Sabah, Malaysia, and Palawan, the Philippines [42]. The most recent study demonstrated that patients with $P$. knowlesi infection were work-related, including Armed Forces and security personnel, boatmen and researchers, Department of Forestry officers, border patrol officers, and non-work-related, including peri-domestic transmission [43].

Comparing patients with severe and non-severe $P$. knowlesi malaria, the present study demonstrated that patients with severe $P$. knowlesi malaria had a higher leucocyte count and parasitaemia level than those with non-severe $P$. knowlesi malaria. The subgroup analysis of study sites demonstrated that a significantly higher leucocyte count was found in the included studies conducted in Sawarak but not in the included studies conducted in Sabah. These results demonstrated that P. knowlesi patients in Sawarak had an immune response that was strongly correlated to $P$. knowlesi infection, as the leucocyte differential count and neutrophil counts were strongly correlated with $P$. knowlesi parasitaemia [23]. The results from a previous study reported that more than $80 \%$ of patients with $P$. knowlesi malaria had a parasitaemia level greater than 100000 parasites/ $\mu \mathrm{l}$ [28]. They also indicated that neutrophil counts were correlated with $P$. knowlesi parasitaemia levels, suggesting that severe $P$. knowlesi malaria can lead to parasite-induced inflammation such as lung injury [28]. The present study found a lower platelet count in patients with severe $P$. knowlesi malaria than in those with non-severe $P$. knowlesi malaria. A previous study reported thrombocytopenia (84.9\%) among patients with $P$. falciparum and $P$. vivax infections [44]. The thrombocytopenia in the present study might be related to a high parasitaemia level, as presented by a study in Thailand [45]. A previous study reported that patients with $P$. knowlesi malaria demonstrated a decrease in coagulation system markers [46] or an increase in the proportion of platelet binding to infected red cells [47]. The risk factors for death from $P$. knowlesi infection demonstrated in Sabah, Malaysia, were female sex, age $\geq 45$ years, cardiovascular disease, microscopic misdiagnosis, and delay in administrating intravenous treatment [48].

The results of the present study demonstrated that severe complications in patients with $P$. knowlesi malaria were higher than those with $P$. falciparum malaria. This result supported the conclusion of a previous study in Sabah, Malaysia, which demonstrated severe P. knowlesi malaria at $29 \%$ with a greater than 3 -fold risk of severity when compared to P. falciparum malaria [28]. Comparing patients with severe $P$. knowlesi and severe P. falciparum malaria, patients with severe $P$. knowlesi infections 
were older than patients with severe $P$. falciparum malaria. This result was similar to a previous study demonstrating that patients with $P$. knowlesi malaria were older than those with P. falciparum or P. vivax malaria [28]. Previous studies have suggested that severe $P$. knowlesi infections and death have not been reported in children, unlike severe $P$. falciparum infections [31].

The present study demonstrated that patients with severe $P$. knowlesi malaria had a higher neutrophil count than patients with severe $P$. falciparum malaria. These results were supported by a previous study that revealed that neutrophil counts strongly correlated with $P$. knowlesi parasitaemia [23]. They suggested that the strong association between $P$. knowlesi parasitaemia and neutrophils involved the cytokines IL-1ra and IL-10, which were related to anti-inflammatory effects on the patient's response to infection [23]. A previous study demonstrated a high neutrophil count with a high neutrophil/lymphocyte (NL) ratio, demonstrating that neutrophil and lymphocyte counts were the most important leucocyte changes during malaria infections [45]. A high neutrophil count among patients with $P$. knowlesi malaria might be due to a consistent correlation between high neutrophil counts and high parasite densities in $P$. falciparum-infected patients when compared to those with low and moderate parasitaemia groups $[45,49]$. Previous studies also demonstrated that the median neutrophil count on admission was higher in severe $P$. knowlesi than in non-severe P. knowlesi infections [23, 27]. The present study demonstrated that patients with severe $P$. knowlesi malaria had a higher creatinine level than those with severe $P$. falciparum malaria. These results demonstrated the association between a higher creatinine level and a higher proportion of severe AKI among patients with severe $P$. knowlesi malaria. As the risk of severity occurred in a high proportion compared to $P$. falciparum malaria in Malaysia, all patients recognized with severe malaria due to $P$. knowlesi were given intravenous artesunate as a first-line treatment upon admission to reduce the potential of mortality [28].

The present study had limitations. First, only a few articles on severe P. knowlesi infections could be selected and extracted, so the pooled analysis needs to be carefully interpreted. Second, some clinical and laboratory data were missing among the seven included studies, causing a low statistical power to infer the differences between severe $P$. knowlesi and severe $P$. falciparum malaria. Third, subgroup analysis of study sites between Sabah and Sarawak in the meta-analysis of severe $P$. knowlesi and severe P. falciparum infections could not be performed, as three studies included in the analysis were conducted in Sabah and only one included study was conducted in Sarawak. Fourth, some confounding factors might have affected the haematological analysis of the present study, such as demographic factors, malaria immunity, underlying diseases, and the haemoglobinopathy of patients with malaria. Due to the limitations of the data on haemoglobinopathies and G6PD deficiency of the study populations reported in the included studies, the effect of haemoglobinopathies and G6PD deficiency on the severity or laboratory parameters between patients with severe $P$. knowlesi and non-severe $P$. knowlesi infections could not be examined in the present study.

\section{Conclusions}

This systematic review demonstrated a high rate of severe $P$. knowlesi infections. Patients with severe P. knowlesi malaria were older, had a higher leucocyte count, and had a lower platelet count than those with nonsevere P. knowlesi malaria. Patients with severe P. knowlesi infections were older, had a higher neutrophil count, and had a higher creatinine level than those with severe P. falciparum infections. Future work must aim to explore the risk factors with a higher number of enrolled studies to aid in the understanding of severe P. knowlesi infections in malaria-endemic settings.

\section{Supplementary information}

Supplementary information accompanies this paper at https://doi.org/10. 1186/s40249-020-00727-x.

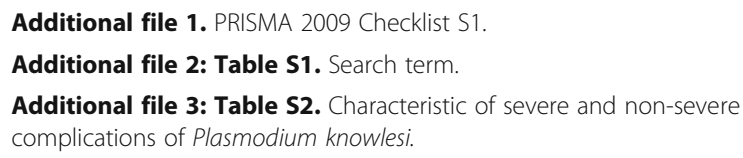

Additional file 4: Table S3. Characteristic of severe Plasmodium knowlesi and P. falciparum.

Additional file 5: Figure S1. The proportion of farmers infected with Plasmodium knowlesi and P. falciparum.

Additional file 6: Figure S2. The proportion of plantation workers infected with Plasmodium knowlesi and P. falciparum.

\section{Abbreviations \\ AKI: Acute kidney injuries; ALT: Alanine aminotransferase; AST: Aspartate aminotransferase; Cl: Confidence interval; MD: Mean difference; NOS: Newcastle-Ottawa Scale; PCR: Polymerase chain reaction; PICO: Population, intervention, control, and outcomes; PRISMA: Preferred Reporting Items for Systematic Review and Meta-Analysis; RDTs: Rapid diagnostic tests; WHO: World Health Organization}

\section{Acknowledgements}

The authors would like to thank all published research that contributed to the data used in this study. The authors are also grateful to Mr. David C. Chang for editing the grammar of this manuscript.

\section{Authors' contributions}

MK carried out the study design, study selection, data extraction, and statistical analysis and drafted the manuscript. KUK participated in the study selection and data extraction and drafted the manuscript. GDM and FRM participated in the study selection and writing of the paper. All authors read and approved the final manuscript. 


\section{Funding}

This research was partially supported by the New Strategic Research (P2P) project, Walailak University, Thailand. The funders had a role in the collection, analysis, and interpretation of the data.

\section{Availability of data and materials}

All data related to the present study are available in the manuscript and supplementary files.

\section{Ethics approval and consent to participate}

Not applicable.

\section{Consent for publication}

Not applicable.

\section{Competing interests}

The authors declare that there is no conflict of interest regarding the publication of this article.

\section{Author details}

${ }^{1}$ Medical Technology, School of Allied Health Sciences, Walailak University, Tha Sala, Nakhon Si Thammarat, Thailand. ${ }^{2}$ Department of Medical Technology, Far Eastern University, Manila, Philippines.

Received: 21 April 2020 Accepted: 14 July 2020

Published online: 29 July 2020

\section{References}

1. Garnham P. Malaria parasites and other haemosporidia. Oxford: Blackwell Scientific; 1966.

2. Singh B, Kim Sung L, Matusop A, Radhakrishnan A, Shamsul SS, Cox-Singh J, et al. A large focus of naturally acquired Plasmodium knowlesi infections in human beings. Lancet. 2004;363(9414):1017-24.

3. Knowles R, Gupta BMD. A study of monkey-malaria, and its experimental transmission to man. Ind Med Gaz. 1932;67(6):301-20.

4. Singh B, Daneshvar C. Human infections and detection of Plasmodium knowlesi. Clin Microbiol Rev. 2013;26(2):165-84.

5. Erratum. A study of monkey-malaria, and its experimental transmission to man. Ind Med Gaz. 1932;67(12):701.

6. Moyes CL, Shearer FM, Huang Z, Wiebe A, Gibson HS, Nijman V, et al. Predicting the geographical distributions of the macaque hosts and mosquito vectors of Plasmodium knowlesi malaria in forested and nonforested areas. Parasit Vectors. 2016;9:242.

7. Sallum MAMPE, Harrison BA, Wilkerson RC. Revision of the Leucosphyrus group of Anopheles (Cellia) (Diptera, Culicidae). Rev Bras Entomol. 2005;49:1-152.

8. Imai N, White MT, Ghani AC, Drakeley CJ. Transmission and control of Plasmodium knowlesi: a mathematical modelling study. PLoS Negl Trop Dis. 2014:8(7):e2978

9. Mulligan Sa. Plasmodium knowlesi. Maryland: National Institute of Allergy and Infectious Diseases; 1932

10. William T, Jelip J, Menon J, Anderios F, Mohammad R, Awang Mohammad TA, et al. Changing epidemiology of malaria in Sabah, Malaysia: increasing incidence of Plasmodium knowlesi. Malar J. 2014:13:390.

11. Barber BE, William T, Grigg MJ, Piera K, Yeo TW, Anstey NM. Evaluation of the sensitivity of a pLDH-based and an aldolase-based rapid diagnostic test for diagnosis of uncomplicated and severe malaria caused by PCRconfirmed Plasmodium knowlesi, Plasmodium falciparum, and Plasmodium vivax. J Clin Microbiol. 2013;51(4):1118-23.

12. Grigg MJ, William T, Barber BE, Parameswaran U, Bird E, Piera K, et al. Combining parasite lactate dehydrogenase-based and histidine-rich protein 2-based rapid tests to improve specificity for diagnosis of malaria due to Plasmodium knowlesi and other Plasmodium species in Sabah, Malaysia. J Clin Microbiol. 2014:52(6):2053-60.

13. Cox-Singh J, Davis TM, Lee KS, Shamsul SS, Matusop A, Ratnam S, et al. Plasmodium knowlesi malaria in humans is widely distributed and potentially life threatening. Clin Infect Dis. 2008:46(2):165-71.

14. WHO. World Malaria Report 2010 2010. Available from: https://www.who.int/ malaria/world malaria report 2010/en/.

15. WHO. World Malaria Report 2011. 2011.

16. WHO. World Malaria Report 2012 2012. Available from: https://www.who.int/ malaria/publications/world_malaria_report_2012/en/.
17. WHO. World Malaria Report 2013 2013. Available from: https://www.who.int/ malaria/publications/world_malaria_report_2013/en/.

18. WHO. World Malaria Report 2014 2014. Available from: https://www.who.int/ malaria/publications/world_malaria_report_2014/en/.

19. WHO. Severe malaria. Geneva: WHO; 2012. Available from: https://apps.who. int/iris/bitstream/handle/10665/79317/9789241548526_eng.pdf;jsessionid= 61F229D7C974C09AE1273C6588FC1ABD?sequence=1.

20. Lo CKMD, Loeb M. Newcastle-Ottawa scale: comparing reviewers' to authors' assessments. BMC Med Res Methodol. 2014;14:45.

21. Nyaga VN, Arbyn M, Aerts M. MetaproPa Stata command to perform metaanalysis of binomial data. Arch Public Health. 2014;72(1):39.

22. Barber BE, Grigg MJ, William T, Piera KA, Boyle MJ, Yeo TW, et al. Effects of aging on parasite biomass, inflammation, endothelial activation, microvascular dysfunction and disease severity in Plasmodium knowlesi and Plasmodium falciparum malaria. J Infect Dis. 2017:215(12):1908-17.

23. Cox-Singh J, Singh B, Daneshvar C, Planche T, Parker-Williams J, Krishna S. Anti-inflammatory cytokines predominate in acute human Plasmodium knowlesi infections. PLoS One. 2011;6(6):e20541.

24. Daneshvar C, Davis TME, Cox-Singh J, Rafa'ee MZ, Zakaria SK, Divis PCS, et al. Clinical and laboratory features of human Plasmodium knowlesi infection. Clin Infect Dis. 2009:49(6):852-60.

25. Grigg MJ, Cox J, William T, Jelip J, Fornace KM, Brock PM, et al. Individuallevel factors associated with the risk of acquiring human Plasmodium knowlesi malaria in Malaysia: a case-control study. Lancet Planet Health. 2017;1(3):e97-e104.

26. William T, Menon J, Rajahram G, Chan L, Ma G, Donaldson S, et al. Severe Plasmodium knowlesi malaria in a tertiary care hospital, Sabah, Malaysia. Emerg Infect Dis. 2011;17(7):1248-55.

27. Willmann M, Ahmed A, Siner A, Wong IT, Woon LC, Singh B, et al. Laboratory markers of disease severity in Plasmodium knowlesi infection: a case control study. Malar J. 2012;11:363

28. Barber BE, William T, Grigg MJ, Menon J, Auburn S, Marfurt J, et al. A prospective comparative study of knowlesi, falciparum, and vivax malaria in Sabah, Malaysia: high proportion with severe disease from Plasmodium knowlesi and Plasmodium vivax but no mortality with early referral and artesunate therapy. Clin Infect Dis. 2013:56(3):383-97.

29. Grigg MJ, William T, Barber BE, Rajahram GS, Menon J, Schimann E, et al. Age-related clinical spectrum of Plasmodium knowlesi malaria and predictors of severity. Clin Infect Dis. 2018;67(3):350-9.

30. Cochrane handbook for systematic reviews of interventions version 6.0 (updated July 2019). Cochrane; 2019. Available from: www.training.cochrane. org/handbook.

31. Rajahram GS, Barber BE, William T, Grigg MJ, Menon J, Yeo TW, et al. Falling Plasmodium knowlesi malaria death rate among adults despite rising incidence, Sabah, Malaysia, 2010-2014. Emerg Infect Dis. 2016;22(1):41-8.

32. Yusof R, Lau YL, Mahmud R, Fong MY, Jelip J, Ngian HU, et al. High proportion of knowlesi malaria in recent malaria cases in Malaysia. Malar J. 2014;13:168

33. Piera KA, Aziz A, William T, Bell D, Gonzalez IJ, Barber BE, et al. Detection of Plasmodium knowlesi, Plasmodium falciparum and Plasmodium vivax using loop-mediated isothermal amplification (LAMP) in a co-endemic area in Malaysia. Malar J. 2017;16(1):29.

34. Hussin N, Lim YA, Goh PP, William T, Jelip J, Mudin RN. Updates on malaria incidence and profile in Malaysia from 2013 to 2017. Malar J. 2020;19(1):55.

35. Gamalo LE, Dimalibot J, Kadir KA, Singh B, Paller VG. Plasmodium knowlesi and other malaria parasites in long-tailed macaques from the Philippines. Malar J. 2019:18(1):147.

36. Imwong M, Madmanee W, Suwannasin K, Kunasol C, Peto TJ, Tripura R, et al. Asymptomatic natural human infections with the simian malaria parasites Plasmodium cynomolgi and Plasmodium knowlesi. J Infect Dis. 2019;219(5):695-702.

37. Barber BE, Grigg MJ, William T, Yeo TW, Anstey NM. The treatment of Plasmodium knowlesi malaria. Trends Parasitol. 2017:33(3):242-53.

38. Daneshvar C, Davis TM, Cox-Singh J, Rafa'ee MZ, Zakaria SK, Divis PC, et al Clinical and parasitological response to oral chloroquine and primaquine in uncomplicated human Plasmodium knowlesi infections. Malar J. 2010;9:238.

39. Nguansangiam S, Day NP. Hien TT, Mai NT, Chaisri U, Riganti M, et al. A quantitative ultrastructural study of renal pathology in fatal Plasmodium falciparum malaria. Tropical Med Int Health. 2007:12(9):1037-50.

40. Dondorp AM, Lee SJ, Faiz MA, Mishra S, Price R, Tjitra E, et al. The relationship between age and the manifestations of and mortality associated with severe malaria. Clin Infect Dis. 2008;47(2):151-7. 
41. Barber BE, William T, Dhararaj P, Anderios F, Grigg MJ, Yeo TW, et al. Epidemiology of Plasmodium knowlesi malaria in north-East Sabah, Malaysia: family clusters and wide age distribution. Malar J. 2012;11:8.

42. Fornace KM, Herman LS, Abidin TR, Chua TH, Daim S, Lorenzo PJ, et al. Exposure and infection to Plasmodium knowlesi in case study communities in northern Sabah, Malaysia and Palawan, The Philippines. PLoS Neglect Trop Dis. 2018;12(6):16.

43. Koh GJ, Ismail PK, Koh D. Occupationally acquired Plasmodium knowlesi malaria in Brunei Darussalam. Saf Health Work. 2019;10(1):122-4.

44. Kotepui M, Phunphuech B, Phiwklam N, Chupeerach C, Duangmano S. Effect of malarial infection on haematological parameters in population near Thailand-Myanmar border. Malar J. 2014;13:218.

45. Kotepui M, Piwkham D, PhunPhuech B, Phiwklam N, Chupeerach C, Duangmano S. Effects of malaria parasite density on blood cell parameters. PLoS One. 2015:10(3):e0121057.

46. Nowak SP, Zmora P, Pielok L, Kuszel L, Kierzek R, Stefaniak J, et al. Case of Plasmodium knowlesi malaria in Poland linked to travel in Southeast Asia. Emerg Infect Dis. 2019;25(9):1772-3.

47. Kho S, Barber BE, Johar E, Andries B, Poespoprodjo JR, Kenangalem E, et al, Platelets kill circulating parasites of all major Plasmodium species in human malaria. Blood. 2018;132(12):1332-44.

48. Rajahram GS, Cooper DJ, William T, Grigg MJ, Anstey NM, Barber BE. Deaths from Plasmodium knowlesi malaria: case series and systematic review. Clin Infect Dis. 2019;69(10):1703-11.

49. McKenzie FE, Prudhomme WA, Magill AJ, Forney JR, Permpanich B, Lucas C, et al. White blood cell counts and malaria. J Infect Dis. 2005;192(2):323-30.

Ready to submit your research? Choose BMC and benefit from:

- fast, convenient online submission

- thorough peer review by experienced researchers in your field

- rapid publication on acceptance

- support for research data, including large and complex data types

- gold Open Access which fosters wider collaboration and increased citations

- maximum visibility for your research: over $100 \mathrm{M}$ website views per year

At $\mathrm{BMC}$, research is always in progress.

Learn more biomedcentral.com/submissions 